\title{
Effect of a cracked surface of porous silicon on the behaviour of the acoustic signature
}

\author{
Samia Bouhedja ${ }^{1, \mathrm{a}}$ and Farah Hamdi ${ }^{2}$ \\ ${ }^{1}$ Faculty of Medical Sciences, University of Constantine3, BP. 125, Chalet des Pins, DZ-25000, \\ Constantine, Algeria \\ ${ }^{2}$ Laboratory LHS, Faculty of Technology Sciences, University of Constantine1, Algeria
}

\begin{abstract}
We study in this work the effect of a crack, located on the porous silicon, Psi, surface on the propagation of Rayleigh waves. We simulate and analyse the acoustic signature $\mathrm{V}(\mathrm{z})$ according porosity at $142 \mathrm{MHz}$, to study the microstructure of PSi around the crack.
\end{abstract}

\section{Introduction}

The determination of fundamental parameters characterizing mechanical properties of materials, by dynamical methods, is based on the propagation of elastic waves in the solids. Our study determines the porous silicon elastic parameters in the case of a cracked surface by using acoustic microscopy. This last wich is a key technique in qualitative and quantitative measures [1,2], is based on the allure of the oscillatory curve of output signal and this is known in literature as the acoustic signature, $\mathrm{V}(\mathrm{z})$. So to study the effect of crack [3], located on the porous silicon surface, on the propagation of Rayleigh waves, we first apply the Schoch model [4,5] to calculate the variation of the reflection coefficient modulus and second the Sheppard-Wilson model in order to simulate and then analyze V (z) by using the fast Fourier transform (F.F.T) spectrum. We are interested, in this work at the most dominant mode appearing in plots of V (z) and which is due to a packet of axial wave interference and Rayleigh waves excited at a given angle of incidence.

\section{Methdology}

In the theory of acoustic microscopy, the angular spectral model is the most widely used to calculate the acoustic materials signature which is written as:

$$
V(Z)=\int_{0}^{\pi / 2} P(\theta)^{2} R(k x)^{2} \sin \theta \cos \theta \exp (2 i(K z Z-K x x) \cos \theta) d \vartheta .
$$

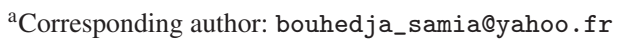

This is an Open Access article distributed under the terms of the Creative Commons Attribution License 4.0, which permits unrestricted use, distribution, and reproduction in any medium, provided the original work is properly cited. 


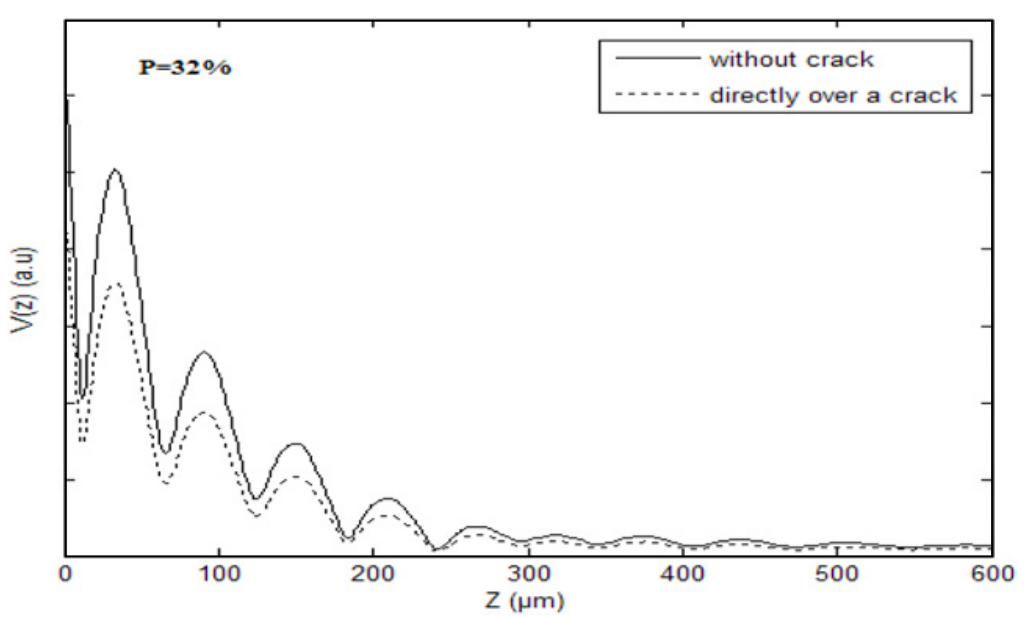

Figure 1. Evolution of the acoustic signature $\mathrm{V}(\mathrm{z})$ for $\mathrm{H}_{2} \mathrm{O} / \mathrm{PSi}$ structure.

Where $\mathrm{P}(\theta)$ is the pupil function of the lens, $\theta$ is the angle between the wave vector $\mathrm{k}$ and the lens axis following $\mathrm{z}$ defocus. $\mathrm{R}\left(\mathrm{k}_{x}\right)$, the reflection coefficient as a function of wave vector $\mathrm{k}$ is becomes in the Schoch model as:

$$
R(k x)=\frac{\left[\left(2 k_{x}^{2}-k_{T}^{2}\right)^{2}-4 k_{x}^{2} \sqrt{\left(k_{x}^{2}-k_{L}^{2}\right)\left(k_{x}^{2}-k_{T}^{2}\right)}\right]-i k_{T}^{4} \sqrt{\left(k_{x}^{2}-k_{L}^{2}\right)} /\left(k^{2}-k_{x}^{2}\right) * \rho_{1} / \rho_{0}}{\left[\left(2 k_{x}^{2}-k_{T}^{2}\right)^{2}-4 k_{x}^{2} \sqrt{\left(k_{x}^{2}-k_{L}^{2}\right)\left(k_{x}^{2}-k_{T}^{2}\right)}\right]+i k_{T}^{4} \sqrt{\left(k_{x}^{2}-k_{L}^{2}\right) /\left(k^{2}-k_{x}^{2}\right)} * \rho_{1} / \rho_{0}} .
$$

With $\mathrm{k}_{L}$ and $\mathrm{k}_{T}$ are respectively the longitudinal and shear wave numbers, $\mathrm{k}_{x}$ is the tangential component of wave vector, $\rho_{l}$ and $\rho_{0}$ are respectively the fluid and solid densities.

\section{Results and discussion}

We carry out calculations in normal conditions of simulation of a scanning mode of reflection acoustic microscope with a working frequency equal to $142 \mathrm{MHz}$. The decrease in the amplitude of the output signal is shown by Fig. 1.

\section{Conclusions}

The modulus of the reflection coefficient decrease is important around the critical excitation angle of surface waves. This is due to the increase of the porosity and the presence of the crack.

The decrease in the amplitude of the output signal is about $29.3 \%$ calculated at critical excitation of Rayleigh wave angle $\theta_{\mathrm{R}}$, and shows the result of the displacement caused by Schoch model in the reflection modulus in the presence of a crack.

\section{References}

[1] A. Briggs, Acoustic Microscopy (2nd ed. Oxford: Clarendon, 2010)

[2] P. Zinin, M. H. Manghnani, Y. C. Wang and R. A. Livingston, NDT\&E International. 33(5), 283287 (2000) 
[3] J. M. Rowe, J. Kushibiki, M. G. Somekh, G. A. D. Briggs, Phil. Trans. R. Soc. Lond. A. 320, 201-214 (1986)

[4] Y. Bouzidi, D. R. Schmitt, IEEE Trans. Ulrason Ferroelectr Freq Control. 55 (2008)

[5] Da Fonsesa R. J. M., Saurel J. M., Foucaran A., Appli. Surf. Sci. (1995) 